\title{
Characteristics of high-frequency emission of an electron in graphene in a constant electric field
}

\author{
I. N. Aslyamova', S. P. Gavrilov $₫ 1,2$ \\ ${ }^{1}$ Herzen State Pedagogical University of Russia, 48 Moika River Emb., Saint Petersburg 191186, Russia \\ 2 Tomsk State University, 36 Lenin Avenue, Tomsk 634050, Russia
}

\begin{abstract}
Authors
Irina N. Aslyamova

Sergey P. Gavrilov, ORCID: 0000-0002-0350-3012, e-mail: gavrilovsergeyp@yahoo.com

For citation: Aslyamova, I. N., Gavrilov, S. P. (2020) Characteristics of high-frequency emission of an electron in graphene in a constant electric field. Physics of Complex Systems, 1 (2), 78-84. DOI: 10.33910/2687-153X-2020-1-2-78-84

Received 1 April 2020; reviewed 25 April 2020; accepted 25 April 2020.

Funding: S.P.G. acknowledges support from Tomsk State University Competitiveness Improvement Program and the partial support from the Russian Foundation for Basic Research (RFBR), Project No. 18-02-00149.

Copyright: (c) The Authors (2020). Published by Herzen State Pedagogical University of Russia. Open access under CC BY-NC License 4.0.

Abstract. The article describes the photon emission of the low-energy electron of a graphene monolayer in a constant electric field that exists during a macroscopic time period. We work in the Fock space representation of the Dirac model, which takes exact account of the effects of vacuum instability caused by an electric field, and in which the interaction between electrons and photons is taken into account in the first-order approximation. We find the main contribution to the total probability of a high-frequency photon emission of the electron and analise angular distribution of the emission.
\end{abstract}

Keywords: graphene monolayer, Fock space representation, high-frequency photon emission.

\section{Introduction}

Low-energy single-electron dynamics in graphene monolayers at the charge neutrality point is described by the Dirac model, being a $2+1$ dimensional version of massless quantum electrodynamisc (QED) with the Fermi velocity $v_{F} \simeq 10^{6} \mathrm{~m} / \mathrm{s}$ playing the role of the speed of light in relativistic particle dynamics. This area is currently under intense development, see the reviews (Sarma, Adam, Hwang, Rossi 2011; Vafek, Vishwanath 2014). In particular, the effect of particle production from vacuum due to a low-frequency electric field is crucial for understanding the conductivity of graphene, especially in the so-called nonlinear regime; e.g., see Ref. (Gavrilov, Gitman, Yokomizo 2012) for the review. Electron-hole pair production at the charge neutrality point (which is an analog of the electron-positron pair production from the vacuum) was recently observed in graphene by its indirect influence on the graphene conductivity, see Ref. (Vandecasteele, Barreiro, Lazzeri et al. 2010).

In this article we consider an infinite flat graphene sample on which a uniform electric field is applied, directed along the axis $x$ on the plane of the sample. We assume that the applied field is a constant electric field that exists during a macroscopically large time period $T$ (we call it as the $T$-constant ) compared to the characteristic time scale $\Delta \mathrm{t}_{\mathrm{st}}=\left(\mathrm{e}|\mathrm{E}| \mathrm{v}_{\mathrm{F}} / \hbar\right)^{-1 / 2} \gg 0.24$ fst specific to graphene, $10^{-12} \mathrm{~s} \| \mathrm{T}>\Delta \mathrm{t}_{\mathrm{st}}$; see Ref. (Gavrilov, Gitman, Yokomizo 2012) for details. This field turns on to $E$ at $-T / 2=t_{\text {in }}$ and turns off to 0 at. $T / 2=t_{\text {out }}$.

The electromagnetic field is not confined to the graphene surface, $\mathrm{z}=0$, but rather propagates (with the speed of light $c$ ) in the ambient $3+1$ dimensional space-time, where $z$ is the coordinate of the axis normal to the graphene plane. The article focuses on the characteristics of high-frequency emission 
$\left(\omega \gg T^{-1}\right)$ of an electron graphene in a constant electric field. Note that such an emission could be observable which makes it highly interesting. Taking into account that a low-frequency external electric field requires a non-perturbative treatment of massless carriers, we use non-perturbative methods of the strong-field QED with unstable vacuum, see Ref. (Fradkin, Gitman, Shvartsman 1991). We work in the Fock space representation of the Dirac model, which takes exact account of the effects of vacuum instability caused by external electric fields, and in which the interaction between electrons and photons is taken into account perturbatively in the first-order approximation, following the general theory (the generalized Furry representation), see Ref. (Fradkin, Gitman, Shvartsman 1991).We use the representation of the Dirac model in the Fock space adjusted for the interaction of photons with Dirac fermions, Ref. (Gavrilov, Gitman 2017). Applying this representation we find the main contribution to the total probability of a high-frequency photon emission by an electron in graphene and analise angular distribution of the emission.

\section{The total probability of a photon emission}

In what follows, we use boldface symbols for three-dimensional vectors and symbols with arrows for in-plane comonents, for example, $\bar{r}=(x, y)$. The Dirac equation in an external field that couples minimally to the electrons on the graphene plane is

$$
\begin{gathered}
i \hbar \partial_{t} \psi(t, \bar{r})=H^{\mathrm{ext}} \psi(t, \bar{r}), \\
H^{\mathrm{ext}}=v_{F} \gamma^{0} \bar{\gamma} \cdot\left(\bar{p}-\frac{e}{c} \overleftarrow{A}^{\mathrm{ext}}(t, \mathbf{r})\right), \quad p^{j}=-i \hbar \partial_{j},
\end{gathered}
$$

where $\psi(t, \overleftarrow{r})$ is a two-component spinorial field, $\bar{\gamma} \cdot \bar{p}=\gamma^{1} p_{x}+\gamma^{2} p_{y}$, the $\gamma$-matrices satisfy the standard anti-comutation relations, $\left\{\gamma^{\mu}, \gamma^{\nu}\right\}=2 \eta^{\mu \nu}, \eta_{\mu v}=\operatorname{diag}(+1-1,-1), \mu, \nu=0,1,2$, and $e$ is the charge of the electron, $e=-|e|$. The algebra of $\gamma$-matrices has two inequivalent representations in 2+1-dimensions, and a distinct (pseudo spin) representation is associated with each Dirac point,

$$
\gamma^{0}=\sigma^{3}, \quad \gamma^{1}=i \sigma^{2}, \gamma^{2}=-i \zeta \sigma^{1},
$$

where the $\sigma^{j}$ are Pauli matrices, and $\zeta= \pm 1$ labels inequivalent representations.

In the usual dipole approximation, $z$-dependence of the QED Hamiltonian can be integrated out. Then the Hamiltonian of the electron-photon interaction is

$$
\begin{aligned}
& \hat{\mathcal{H}}_{\text {int }} \approx-\left.\int \bar{\jmath}_{\text {in }}(t, \bar{r}) \cdot \overline{\hat{A}}(t, \mathbf{r})\right|_{z=0} d \bar{r}, \\
& \bar{j}_{\text {in }}(t, \bar{r})=\frac{e v_{F}}{2 c}\left[\hat{\Psi}^{\dagger}(t, \bar{r}), \gamma^{0} \bar{\gamma} \hat{\Psi}(t, \bar{r})\right]_{-},
\end{aligned}
$$

where quantum fields $\hat{\emptyset}(t, \bar{r})$ and $\hat{\emptyset}^{\dagger}(t, \bar{r})$ obey both the Dirac equation with the potential $\bar{A}^{\text {ext }}(t, \bar{r})$ and the standard equal time anticommutation relations. We decomposed quantum electromagnetic field in the interaction representation into terms of the annihilation and creation operators of photons, $C_{\mathbf{k} \vartheta}$ and $C_{\mathbf{k} \vartheta}^{\dagger}$ :

$$
\hat{\mathbf{A}}(t, \mathbf{r})=c \sum_{\mathbf{k}, \vartheta} \sqrt{\frac{2 \pi \hbar}{\varepsilon V \omega}} \stackrel{a}{\mathbf{k}} \vartheta\left[C_{\mathbf{k} \vartheta} \mathrm{e}^{i(\mathbf{k} \cdot \mathbf{r}-\omega t)}+C_{\mathbf{k} \vartheta}^{\dagger} \mathrm{e}^{-i(\mathbf{k} \cdot \mathbf{r}-\omega t)}\right],
$$

where $\vartheta=1,2$ is a polarization index, the $\varepsilon_{\mathrm{k} 9}$ are unit polarization vectors transversal to each other and to the wavevector $\mathbf{k}, \omega=c k, \mathbf{k}=|\mathbf{k}|, V$ is the volume of the box regularization, and $\varepsilon$ is the relative permittivity ( $\varepsilon=1$ for graphene suspended in vacuum).

The in - and out - operators of creation and annihilation of electrons $\left(a_{n}^{\dagger}, a_{n}\right)$ and holes $\left(b_{n}^{\dagger}, b_{n}\right)$ are defined by the two representations of the quantum Dirac field $\hat{\Psi}(t, \bar{r})$ as

$$
\begin{aligned}
& \hat{\Psi}(t, \bar{r})=\sum_{n}\left[a_{n}(\text { in })_{+} \psi_{n}(t, \bar{r})+b_{n}^{\dagger}\left(\text { in }{ }_{-} \psi_{n}(t, \bar{r})\right]\right. \\
& \left.=\sum_{n}\left[a_{n}(\text { out }){ }^{+} \psi_{n}(t, \bar{r})+b_{n}^{\dagger}(\text { out })\right)^{-} \psi_{n}(t, \bar{r})\right],
\end{aligned}
$$


where ${ }_{\zeta} \psi_{n}(t, \overleftarrow{r})$ and ${ }^{\zeta} \psi_{n}(t, \bar{r})$ are in - and out -solutions of the Dirac equation with the potential $\bar{A}^{\text {ext }}(t, \bar{r})$ for given quantum numbers $n$ and well-defined sign of the frequency $\zeta$ either before the turnon or after the turn-off of the field, respectively. They are related by a linear transformation, i.e. a linear canonical transformation (the Bogoliubov transformation) between in - and out - operators; see (Fradkin, Gitman, Shvartsman 1991) for details.

The initial and final states with definite numbers of charged particles and photons can be generally written in the following way:

$$
\begin{aligned}
& \left.\mid \text { in }\rangle=C^{\dagger} \ldots b^{\dagger}(\text { in }) \ldots a^{\dagger}(\text { in }) \ldots \mid 0, \text { in }\right\rangle, \\
& \left.\mid \text { out }\rangle=C^{\dagger} \ldots b^{\dagger} \text { (out) } \ldots a^{\dagger} \text { (out) } \ldots \mid 0, \text { out }\right\rangle .
\end{aligned}
$$

The $\mathcal{S}$-matrix or the scattering operator in the first-order approximation with respect of electronphoton interaction (it is exact with respect of an interaction with an external field) is

$$
\mathcal{S} \approx 1+i \mathrm{Y}^{(1)}, \quad \mathrm{Y}^{(1)}=-\frac{1}{\hbar} \int_{-\infty}^{\infty} \gamma \hat{\mathcal{H}}_{\text {int }} d t
$$

In general, the emission of a single photon by an electron is accompanied by the creation of a number of electron-hole pairs from the vacuum by the quasiconstant electric field. We are interested in the probability of transition from the single-electron state characterized by the quantum numbers $l$ with the emission of one photon with given $\mathbf{k}, \vartheta$ and the production of arbitrary number of pairs from the vacuum, that is, the total probability of the emission of the given photon from the single-electron state, $\mathcal{P}\left(\mathbf{k} \vartheta||^{+}\right)$. This probability can be presented as the average number of photon emitted

$$
\left.\mathcal{P}\left(\left.\mathbf{k} \vartheta\right|^{+}\right)=0, \text { in } \mid a_{n}(\text { in }) \mathcal{S}^{\dagger} C_{\mathbf{k} \vartheta}^{\dagger} C_{\mathbf{k} \vartheta} \mathcal{S} a_{l}^{\dagger}(\text { in }) \mid 0, \text { in }\right\rangle .
$$

It is convenient to represent Eq. (7) as

$$
\begin{aligned}
& \mathcal{P}\left(\mathbf{k} \vartheta \mid+l^{+}\right)=\sum_{n}\left|w_{i n}^{(1)}\left(\begin{array}{c}
n ; \mathbf{k} \vartheta \mid \\
l
\end{array}\right)\right|^{2}, \\
& \left.w_{i n}^{(1)}(\stackrel{+}{n} ; \mathbf{k} \vartheta \mid \stackrel{+}{l})=0, \text { in } \mid a_{n}(\text { in }) C_{\mathbf{k} \vartheta} i \mathrm{Y}^{(1)} a_{l}^{\dagger}(\text { in }) \mid 0, \text { in }\right\rangle .
\end{aligned}
$$

Note that to calculate the amplitude $w_{i n}^{(1)}\left({ }_{n}^{+} ;\left.\mathbf{k} \vartheta\right|^{+}\right)^{\prime}$ we need only initial electron states.

\section{The case of a constant electric field}

Let us calculate the total probability for emission, given by Eq. (8), in a near constant electric field that is realised as the $T$-constant electric field (Gavrilov, Gitman 2017). We consider the case of the field described by a vector potential with only one nonzero component $A_{1}^{\text {ext }}(t)\left(A_{\mu}^{\text {ext }}(t)=0, \mu \neq 1\right)$,

$$
A_{1}^{\text {ext }}(t)=c E \begin{cases}t_{1} & t \in \mathrm{I}=\left(-\infty, t_{\text {in }}\right), t_{1}=-T / 2, \\ t, & t \in \mathrm{Int}=\left[t_{1}, t_{1}\right], \\ t_{2}, & t \in \mathrm{II}=\left(t_{2}, \infty\right), t_{2}=T / 2\end{cases}
$$
i.e.,

such that the electric field $\mathbf{E}(t)$ also has only one nonzero component, which is nonzero for $t \in \mathrm{Int}$,

$$
E^{1}(t)=E, \quad t \in \mathrm{Int} ; \quad E^{1}(t)=0, \quad t \in \mathrm{I} \cup \mathrm{II} .
$$

To simplify notation, it is convenient to let $E<0$, so that $e E>0$. We are only interested in the case of a slowly varying homogeneous electric field $E$ which remains constant for a macroscopically large time period $T$,

$$
T / \Delta t_{s t} \gg 1 \text {. }
$$


The time scale $\Delta t_{s t}$ plays the role of the stabilization time in the sense that the differential mean numbers of created pairs have the form

$$
N_{n} \simeq e^{-\pi \lambda}, \quad \lambda=\frac{v_{F} p_{y}^{2}}{e E \hbar}
$$

which is the same for the case of the constant electric field in a finite momentum range.

The solutions of the Dirac equation (1) in the $T$-constant field are studied in details in Ref. (Gavrilov, Gitman 2017). It was demonstrated that the in-set $\left\{{ }_{\zeta} \psi_{n}(t, \bar{r})\right\}$ of these solutions can be taken in the form

$$
\begin{gathered}
{ }_{ \pm} \psi_{\vec{p}}(t, \bar{r})=\left(i \hbar \partial_{t}+H^{e x t}\right){ }_{ \pm} \phi_{\vec{p}, \mp 1}(t, \bar{r}), \\
{ }_{ \pm} \phi_{\vec{p}, \mp 1}(t, \bar{r})=\mathrm{e}^{i \vec{p} \cdot \vec{r} / \hbar}{ }_{ \pm} \varphi_{\vec{p}, \mp 1}(t) U_{\mp 1},
\end{gathered}
$$

where $U_{s}$ are the constant orthonormalized spinors

$$
U_{+1}=\frac{1}{\sqrt{2}}\left[\begin{array}{l}
1 \\
1
\end{array}\right], \quad U_{-1}=\frac{1}{\sqrt{2}}\left[\begin{array}{l}
1 \\
-1
\end{array}\right] \text {. }
$$

In a finite momentum range, the functions ${ }_{ \pm} \varphi_{\vec{p}, s}(t)$ take the form of the following Weber parabolic cylinder (WPC) functions (Bateman 1953):

$$
\begin{aligned}
& { }_{+} \varphi_{\vec{p}, s}(t)=C D_{v-\frac{1-s}{2}}[-(1-i) \xi], \quad \varphi_{\vec{p}, s}(t)=C D_{-v-\frac{1+s}{2}}[-(1+i) \xi], \\
& \xi=\sqrt{\frac{v_{F}}{e E \hbar}}\left(e E t+p_{x}\right), \quad v=\frac{i \lambda}{2},
\end{aligned}
$$

with the normalization constant

$$
C=\left(2 e E \hbar v_{F} S\right)^{-1 / 2} \exp (-\pi \lambda / 8)
$$

where $S$ is the graphene area. An in-state ${ }_{ \pm} \psi_{\vec{p}}(t, \bar{r})$ describes a particle/hole with a well-defined energy in the distant past. In such a description, the probability of an emission of a given photon in the $T$-constant electric field for $t \in$ Int is indistinguishable from the effect produced by a constant field $(T \rightarrow \infty)$. Then, in what follows, we assume that $T \rightarrow \infty$.

It is useful to define an orthonormal triple

$$
\begin{aligned}
& \mathbf{k} / k=(\sin \theta \cos \phi, \sin \theta \sin \phi, \cos \theta), \\
& \stackrel{\circ}{\mathbf{k} 1}_{2}=\mathbf{e}_{z} \times \mathbf{k} /\left|\mathbf{e}_{z} \times \mathbf{k}\right|, \quad \stackrel{\circ}{\mathbf{k} 2}_{1}=\mathbf{k} \times \stackrel{\circ}{\mathbf{k} 1}_{1} /|\mathbf{k} \times \stackrel{\circ}{\mathbf{k} 1}|,
\end{aligned}
$$

where

$$
\begin{aligned}
& \stackrel{\circ}{\mathbf{k} 1}=(-\sin \phi, \cos \phi, 0), \\
& \stackrel{\circ}{\mathbf{k} 2}=(-\cos \theta \cos \phi,-\cos \theta \sin \phi, \sin \theta)
\end{aligned}
$$


for $\mathbf{k}$ in the upper spatial region, $k_{z} \geq 0$. Using the parametrization, $d \mathbf{k}=c^{-3} \omega^{2} d \omega d \Omega$, we find that the probabilty of the emission per unit frequency and solid angle $d \Omega$ is

$$
\begin{aligned}
& \frac{d \mathcal{P}(\mathbf{k} \vartheta \mid \stackrel{+}{\bar{p}})}{d \omega d \dot{\mathrm{U}}}=\left.\frac{\alpha}{\varepsilon}\left(\frac{v_{F}}{c}\right)^{2} \frac{\omega \ddot{\mathrm{A}} t_{s t}^{2}}{(2 \pi)^{2}}\left|M_{\bar{p}^{\prime} \bar{p}}^{+}\right|^{2}\right|_{\vec{p}^{\prime}=\bar{p}-\hbar \vec{k}}, \\
& M_{\bar{p}^{\prime} \bar{p}}^{+}=v_{F}^{2} S C^{\prime} C \exp \left(-i \omega \frac{p_{x}+p_{x}^{\prime}}{2 e E}\right) \sqrt{\frac{\hbar}{v_{F} e E}} \\
& \times\left\{p_{y}^{\prime} p_{y} \chi_{\vartheta}^{1,1} Y_{11}+2 \frac{e E \hbar}{v_{F}} \chi_{\vartheta}^{0,0} Y_{00}\right. \\
& \left.+\sqrt{\frac{e E \hbar}{v_{F}}} \zeta\left[(1-i) p_{y}^{\prime} \chi_{\vartheta}^{1,0} Y_{10}+(1+i) p_{y} \chi_{\vartheta}^{0,1} Y_{01}\right]\right\},
\end{aligned}
$$

where $\alpha=\mathrm{e}^{2} / c \ddot{y}$ is the fine structure constant, $C^{\prime}=\left.C\right|_{p_{y} \rightarrow p_{y}^{\prime}}$, and

$$
\begin{aligned}
& \chi_{\vartheta}^{\left(1+s^{\prime}\right) / 2,(1+s) / 2}=U_{s^{\prime}}^{\dagger} \gamma^{0} \bar{\gamma} \cdot \vec{\varepsilon}_{\mathbf{k} \vartheta} U_{s}, \\
& \chi_{1}^{0,0}=-\chi_{1}^{1,1}=\sin \phi, \quad \chi_{1}^{1,0}=-\chi_{1}^{0,1}=i \zeta \cos \phi \\
& \chi_{2}^{0,0}=-\chi_{2}^{1,1}=\cos \theta \cos \phi, \quad \chi_{2}^{1,0}=-\chi_{2}^{0,1}=-i \zeta \cos \theta \sin \phi
\end{aligned}
$$

In the case of a high frequency of interest, $Y_{j^{\prime} j}(\rho)$ can be approximated as the following Fourier transformation of the product of the WPC functions

$$
\begin{gathered}
Y_{j^{\prime} j}(\rho) \simeq \int_{-\infty}^{+\infty} D_{-v^{\prime}-j^{\prime}}[-(1+i) u] D_{v-j}[-(1-i) u] \mathrm{e}^{i \rho u} d u, \\
v=\frac{i \lambda}{2}, \quad v^{\prime}=\frac{i \lambda^{\prime}}{2}, \quad \lambda^{\prime}=\left.\lambda\right|_{p_{y} \rightarrow p_{y}^{\prime}}, \quad \rho \approx \Delta t_{s t} \omega .
\end{gathered}
$$

The integrals of the type (17) have been studied in Ref. (Nikishov 1970). Similarly, one can find that

where

$$
\begin{aligned}
& Y_{j^{\prime} j}(\rho)=\sqrt{\frac{2}{\pi}} \sinh \left(\frac{\pi \lambda}{2}\right) \Gamma\left(i \frac{\lambda}{2}-j\right) \\
& \times \exp \left[-\frac{\pi \lambda^{\prime}}{4}+i \frac{\pi}{2}\left(j^{\prime}+2 j-1\right)\right] I_{j^{\prime} j}(\rho), \\
& I_{j^{\prime} j}(\rho)=\left.I(\rho)\right|_{\substack{v \rightarrow++1-j, v^{\prime} \rightarrow v^{\prime}+j^{\prime}}},
\end{aligned}
$$

$$
\begin{aligned}
& I(\rho)=\sqrt{\pi} \exp \left[-i \frac{\pi}{4}+i \frac{\rho^{2}}{4}+i \frac{\pi}{4}\left(v^{\prime}-v\right)+\left(v-v^{\prime}\right) \ln (\rho / \sqrt{2})\right] \\
& \times \Psi\left(v, 1+v-v^{\prime} ;-i \rho^{2} / 2\right)
\end{aligned}
$$

is expressed via the confluent hypergeometric function $\Psi$ and the gamma function $\Gamma$. 


\section{Characteristics of high-frequency emission}

Assuming typical values of an electric field we obtain the following frequency scale

$$
\begin{aligned}
& E=a E_{0}, \quad E_{0}=1 \times 10^{6} \mathrm{~V} / \mathrm{m}, \quad 7 \times 10^{-4} \ll a \ll 8, \\
& \omega_{s c}=\Delta t_{s t}^{-1} \approx \sqrt{a} \times 0.38 \times 10^{14} \mathrm{~s}^{-1} .
\end{aligned}
$$

At high frequencies, $\rho \gg 1$, we can use the asymptotic behavior of the function $\Psi$, given by Eq. (6.13.1.(1)) in (Bateman 1953),

$$
\Psi\left(a, c ;-i \rho^{2} / 2\right)=e^{i \frac{\pi}{2} a}\left(\rho^{2} / 2\right)^{-a}\left[1+O\left(\rho^{-2}\right)\right] .
$$
The term
whatever $\lambda$,$\left|M_{\dot{p}^{\prime} \bar{p}}^{+}\right|^{2}$, given in Eq. (15), is Gaussian function of $\lambda^{\prime}$. It is exponentially small for $\lambda^{\prime}>1$ and

$$
\left|M_{\bar{p}^{\prime} \bar{p}}^{+}\right|^{2} \sim e^{-\pi \lambda^{\prime}} \sinh \left(\frac{\pi \lambda}{2}\right) \exp \left(-\frac{\pi \lambda}{2}\right) .
$$

Then, in what follows, of interest is the case of small $\lambda^{\prime}>1$ only. It implies an approximate conservation law,

$$
v_{F} k_{y} \omega_{s c}^{-1} \sim \operatorname{sign}\left(p_{y}\right) \sqrt{\lambda}
$$

Under this condition it can be shown that the leading contribution to the probability (15) is from terms with $Y_{00}$ and $Y_{01}$,

$$
\begin{aligned}
& Y_{00}(\rho) \approx f \tilde{\mathrm{A}}\left(i \frac{\lambda}{2}\right) e^{-i \pi / 2}\left(\frac{\rho}{\sqrt{2}}\right)^{-\left(v^{\prime}+v+1\right)}, \\
& Y_{01}(\rho) \approx f \tilde{\mathrm{A}}\left(i \frac{\lambda}{2}-1\right) e^{i \pi / 4}\left(\frac{\rho}{\sqrt{2}}\right)^{-\left(v^{\prime}+v\right)}, \\
& f=\sqrt{2} \sinh \left(\frac{\pi \lambda}{2}\right) \exp \left[i \frac{\rho^{2}}{4}-\frac{3 \pi \lambda^{\prime}}{8}-\frac{\pi \lambda}{8}\right] .
\end{aligned}
$$

Thus, we obtain that

$$
\begin{aligned}
& \left|M_{\bar{p}^{\prime} \bar{p}}^{+}\right|^{2} \approx \frac{\hbar}{v_{F} e E} \frac{2 \pi}{\lambda} \sinh \left(\frac{\pi \lambda}{2}\right) \exp \left[-\pi\left(\lambda^{\prime}+\lambda / 2\right)\right] \\
& \times\left|\chi_{\vartheta}^{0,0} \frac{2}{\rho}-\zeta \operatorname{sign}\left(p_{y}\right) \chi_{\vartheta}^{0,1} \frac{\operatorname{sign}\left(p_{y}\right) \sqrt{\lambda}}{i \frac{\lambda}{2}-1}\right|^{2} .
\end{aligned}
$$

Unlike the example considered in Ref. (Gavrilov, Gitman 2017) under condition $|\lambda-\lambda>|<1$ the ex-

pression given by Eq. (25) is valid at whatever $\lambda>$ and $\lambda$.
At small $\sqrt{\lambda}<2 / \rho$ the term with $\chi_{\vartheta}^{0,0}$ in Eq. (25) is leading. In this case $\left|M_{\bar{p}^{\prime} \bar{p}}^{+}\right|^{2} \sim \rho^{-2}$ is rather
small. small.

The main contribution to Eq. (25) is due to the term with $\chi_{\vartheta}^{0,1}$ when $\rho \gg 1 / \sqrt{\lambda}$,

$$
\left|M_{\bar{p}^{\prime} \bar{p}}^{+}\right|^{2} \approx \frac{\hbar}{v_{F} e E} \frac{2 \pi}{(\lambda / 2)^{2}+1} \sinh \left(\frac{\pi \lambda}{2}\right) \exp \left[-\pi\left(\lambda^{\prime}+\lambda / 2\right)\right]\left|\chi_{\vartheta}^{0,1}\right|^{2} .
$$
We see that the probability of the emission (15) where the factor $\left|M_{\bar{p}^{\prime} \bar{p}}^{+}\right|^{2}$ is given by Eq. (26) has
a maximum at $\lambda \sim 1$. 
In this case, taking into account Eq. (23), we can estimate $k_{p^{2}}$ component as $k_{y} \sim \omega_{s c} / v_{F^{*}}$ An angular distribution for the emission is determined by the factor $\left|\chi_{9}^{0,1}\right|^{2}$ given by Eq. (16). We finally find the emission with a polarization index $\vartheta=1$ tends to its maximum at $\phi \rightarrow 0, \pi$, when $\left|k_{x} / k_{y}\right| \gg 1$ and whatever $k_{z}$. That is, the maximum of the emission is near plane $y=0$. The emission with a polarization index $\vartheta=2$ tends to its maximum at $\phi \rightarrow \pm \pi / 2$ and $\theta \rightarrow 0$, when $k_{z}^{2} \gg k_{x}^{2}+k_{y}^{2}$ and $\left|k_{y} / k_{x}\right| \gg 1$. That is, this emission is directed near plane $x=0$ and concentrated near axis $z$.

\section{References}

Bateman, H. (1953) Higher transcendental functions: In 3 vols. Vol. 1. New York; Toronto; London: McGraw-Hill Book Company, 302 p. (In English)

Fradkin, E. S., Gitman, D. M., Shvartsman, S. M. (1991) Quantum electrodynamics with unstable vacuum. Berlin: Springer, 288 p. (In English)

Gavrilov, S. P., Gitman, D. M. (2017) Radiative processes in graphene and similar nanostructures in strong electric fields. Russian Physics Journal, 59 (11), 1870-1874. DOI: 10.1103/PhysRevD.53.7162 (In English)

Gavrilov, S. P., Gitman, D. M., Yokomizo, N. (2012) Dirac fermions in strong electric field and quantum transport in graphene. Physical Review D, 86 (12), 125022. DOI: 10.1103/PhysRevD.86.125022 (In English)

Nikishov, A. I. (1970) Quantum processes in a constant electric fields. Soviet Physics JETP, 32 (4), 690-694. (In English)

Sarma, S. D., Adam, S., Hwang, E. H., Rossi, E. (2011) Electronic transport in two-dimensional graphene. Reviews of Modern Physics, 83 (2), 407-470. DOI: 10.1103/RevModPhys.83.407 (In English)

Vafek, O., Vishwanath, A. (2014) Dirac fermions in solids: from high-T_c cuprates and graphene to topological insulators and Weyl semimetals. Annual Review of Condensed Matter Physics, 5, 83-112. DOI: 10.1146/annurevconmatphys-031113-133841 (In English)

Vandecasteele, N., Barreiro, A., Lazzeri, M. et al. (2010) Current-voltage characteristics of graphene devices: Interplay between Zener-Klein tunneling and defects. Physical Review B, 82, 045416. DOI: 10.1103/PhysRevB.82.04541 (In English) 